Trauma Berufskrankh $2006 \cdot 8$ [Suppl 1]: S110-S115

DOI 10.1007/s10039-006-1135-x

Online publiziert: 20. April 2006

(c) Springer Medizin Verlag 2006
M. Vogt

Klinik für Unfall- und Wiederherstellungschirurgie, BG-Unfallklinik, Tübingen

\section{Kompetenzzentrum Rehabilitationsabklärung}

\section{Erste Erfahrungen mit dem Modellprojekt}

Das Projekt „Kompetenzzentrum Rehabilitationsabklärung “ des Landesverbands Südwestdeutschland der gewerblichen Berufsgenossenschaften verfolgt das Ziel der Optimierung des Heilverfahrens. Es wird seit September 2004 an den BG-Unfallkliniken Ludwigshafen und Tübingen durchgeführt. In Ergänzung zu den Ausführungen von Herrn Dr. V. Kaiser soll aus ärztlicher Sicht über erste Erfahrungen berichtet werden.

\section{Indikationen zur Anwendung des Verfahrens}

Es handelt sich im Wesentlichen um alle Fälle mit im Lauf der Behandlung oder der beruflichen Wiedereingliederung aufgetretenen Komplikationen oder Schwierigkeiten:

- Überschreitung der „normalen“ Dauer der Arbeitsunfähigkeit („Weller-Tabelle"), wenn z. B. der Wiedereintritt der Arbeitsfähigkeit nicht absehbar ist

- Kein organisches Korrelat für geklagte Beschwerden

- Prüfung arbeits- bzw. arbeitsplatzbezogener Maßnahmen

- Probleme bei Arbeits- und Belastungserprobung

- Komplikationsdiagnosen

a) im Zusammenhang mit der Unfallverletzung (z. B. Morbus Sudeck, Tinnitus)

b) unabhängig von der Unfallverletzung (z. B. Diabetes, Suchtproblematik)

- Prüfung konsiliarischer Untersuchungen (z. B. neurologisch, psychiatrisch)
- Festlegung weiterer Behandlungsmaßnahmen (z. B. EAP, BGSW, Schmerztherapie)

- Abstimmung arbeits- bzw. arbeitsplatzbezogener Maßnahmen

- Feststellung erforderlicher orthopädietechnischer Versorgung

- Vorstellung vor größeren Eingriffen (z. B. TEP im Bereich Hüfte oder Knie)

- schwere neurologisch-traumatologische Fälle (z. B. Querschnittlähmungen)

- Fälle mit Problematiken nach $\$ 46$ Abs. 3 SGB VII (78. Woche)

- Beurteilung Arbeitsunfähigkeit/Erwerbsunfähigkeit

\section{Durchführung}

\section{Fachpersonelle Funktionen}

Durch ein Team, welches regelhaft aus einem die Federführung übernehmenden Unfallchirurgen mit D-Arzt-Qualifikation, einem Psychologen und einem Physiotherapeuten besteht, sollen bestehende Probleme aufgedeckt und Lösungsansätze aufgezeigt werden. Bedarfsweise können hier - in Abhängigkeit von der jeweiligen Problematik - weitere medizinische Fachgebiete mit einbezogen werden; beispielsweise Ärzte für Rehabilitationsmedizin, Arbeitsmedizin, Orthopädie, Neurologie und Psychiatrie, Augen- und HNOÄrzte, Schmerztherapeuten, Ergotherapeuten usw.

Der Unfallchirurg erstellt ein Profil der Verletzungen und deren bisheriger Versorgung sowie der aktuell bestehen- den Folgen. Dieses bildet die Basis zur Einschätzung der weiteren Prognose des Heilverlaufs, wobei das Zeitraster mit zu berücksichtigen ist (• Abb. 1).

Der Psychologe evaluiert die Einstellung und Erwartungshaltung des Verletzten, stellt möglicherweise vorliegende Komorbiditäten fest und schätzt unter besonderer Berücksichtigung von Schmerzverarbeitung und situativen Belastungen die Motivation ein.

Dem Physiotherapeuten obliegt die ausführliche Befundaustestung mit Teilnahme des Patienten auch an Einzel- und Gruppentherapie, isokinetischer Untersuchung sowie Einsatz des Worksimulators und entsprechender Messgeräte. Hieraus entsteht ein aussagekräftiges objektives Bild über bestehende Defizite, aus dem sich schließlich eine Prognose des zu er-

\begin{tabular}{llll}
$\begin{array}{l}\text { Tab. } 1 \text { Auswertung der Rückmeldebö- } \\
\text { gen (25 von 37) }\end{array}$ & + & + - & -- \\
& & & \\
Umsetzung der Emp- & 23 & 1 & 1 \\
fehlungen durch BG & & & \\
\hline $\begin{array}{l}\text { Akzeptanz der Emp- } \\
\text { fehlungen durch } \\
\text { Versicherte }\end{array}$ & 17 & 2 & 6 \\
\hline $\begin{array}{l}\text { Zwischenzeitlich } \\
\text { eingetretene Arbeits- } \\
\text { fähigkeit }\end{array}$ & 14 & & 11 \\
$\begin{array}{l}\text { Hinreichende Infor- } \\
\text { mationen an BG durch }\end{array}$ & 22 & 1 & 2 \\
$\begin{array}{l}\text { Rehabilitationsteam } \\
\text { Einschätzung des } \\
\text { Erfolgs für den Versi- } \\
\text { cherten durch BG }\end{array}$ & 17 & 3 & 4 \\
$\begin{array}{l}\text { Zufriedenheit der BG } \\
\text { mit dem Angebot }\end{array}$ & 23 & 2 & \\
\hline
\end{tabular}


wartenden Behandlungsergebnisses ableiten lässt (• Abb. 2).

Schließlich erfolgen durch dieses Team in einer gemeinsamen Besprechung, an welcher auch der Verletzte und fallweise auch ein Vertreter der Berufsgenossenschaft teilnimmt, eine umfassende Bestandsaufnahme der Verletzungsfolgen und eine Analyse des gegenwärtigen Status. Nach Klärung der Frage weiterer möglicher medizinischer oder auch berufsspezifischer Maßnahmen wird schlussendlich eine Prognose erarbeitet, die sowohl für den Betroffenen als auch den zuständigen Unfallversicherungsträger eine Hilfestellung zur Reintegration bzw. bezüglich weiterer einzuleitender Schritte zur Minimierung verbleibender Unfallfolgen geben soll.

\section{Sächliche Ausstattung}

Das Projekt sieht den vorhandenen technischen Standard der BGSW-Anforderungen, ein Assessment für arbeitsplatzbezogene Maßnahmen (EFL, ERGOS) sowie die Möglichkeiten der CT- und MRTDiagnostik vor.

In den an dem Projekt beteiligten BGUnfallkliniken Ludwigshafen und Tübingen kommt das System EFL („Evaluation der funktionellen Leistungsfähigkeit“ nach Isernhagen) zur Anwendung. Es handelt sich hierbei um ein in den USA entwickeltes System, dem das Prinzip der Untersuchung der Belastbarkeit für häufige physische Funktionen der Arbeitstätigkeit mit 29 standardisierten funktionellen Leistungstests (Heben, Tragen, Überkopfarbeit, Leitersteigen, Handkoordination usw.) zugrunde liegt. Die umfassende Testserie wird mit einer Dauer von etwa 6-8 h an 2 aufeinander folgenden Tagen durchgeführt. Ziel sind eine möglichst realitätsgerechte Beurteilung der Leistungsfähigkeit und eine detaillierte Erfassung der physischen Fähigkeiten bzw. bestehender Defizite zur Planung der weiteren beruflichen Rehabilitation (• Abb. 3).

\section{Prognose}

$\mathrm{Zu}$ einer realistischen und umfassenden Beurteilung werden die kompletten Krankenunterlagen, ein Vorerkrankungenver-

Trauma Berufskrankh 2006 - 8 [Suppl 1]: S110-S115 DOI 10.1007/s10039-006-1135-x

(c) Springer Medizin Verlag 2006

M. Vogt

Kompetenzzentrum Rehabilitationsabklärung. Erste Erfahrungen mit dem Modellprojekt

\section{Zusammenfassung}

Das Projekt des Landesverbands Südwestdeutschland der gewerblichen Berufsgenossenschaften „Kompetenzzentrum Rehabilitationsabklärung" verfolgt das Ziel der Optimierung des Heilverfahrens. Es wird seit September 2004 in den BG-Unfallkliniken Ludwigshafen und Tübingen durchgeführt. Bis September 2005 wurde es bei 90 Patienten angewendet. Dabei war es in der Regel möglich, einen Konsens aller Beteiligten bezüglich des weiteren Verlaufs des Heilverfahrens herbeizuführen, wobei auch meist eine Zustimmung der Verletzten zu erkennen war. Um einen ersten Überblick über die längerfristigen Ergebnisse und die Akzeptanz des neuen Verfahrens bei den Berufsgenossenschaften zu

erhalten, wurden Rückmeldebögen an die Auftraggeber versandt, deren Rücklaufquote lag bei knapp 70\%. Die damit vorliegenden ersten Ergebnisse können als durchaus ermutigend bezeichnet werden: Beurteilung und Akzeptanz des Verfahrens waren sowohl bei den Berufsgenossenschaften als auch bei den Versicherten überwiegend positiv. Auch die beteiligten Kliniken erachten das Verfahren als sinnvoll.

\section{Schlüsselwörter}

Landesverband Südwestdeutschland der gewerblichen Berufsgenossenschaften · Optimierung des Heilverfahrens · Kompetenzzentrum Rehabilitationsabklärung · Beurteilung . Akzeptanz

\section{Centre of excellence for "Rehabilitationsabklärung". Initial experience with the model project}

\section{Abstract}

The project on centres of excellence for "Rehabilitationsabklärung" started by the southwest Germany chapter of the commercial employers' liability insurance associations is directed at optimizing treatment. It has been in progress since September 2004 in the Associations' trauma clinics in Ludwigshafen and Tübingen. Up to September 2005 it had been applied in 90 patients. It was generally possible to reach consensus among all concerned about the further course of treatment, agreement of the injured patient being recognizable in most cases in addition. To obtain an initial overview of the longer term results and the acceptance of the new procedure in the insurance associations, feedback forms were sent to the employers; the return rate was around $70 \%$. The initial results collected from these responses can definitely be classed as encouraging. Assessment and acceptance of the procedure were positive for the most part both among the insurance associations and among the insured parties. The clinics taking part also found the procedure helpful.

\section{Keywords}

Southwest Germany chapter of the commercial employers'liability insurance associations - Optimisation of treatment - Centre of excellence in "Rehabilitationsabklärung" · Assessment $\cdot$ Acceptance 


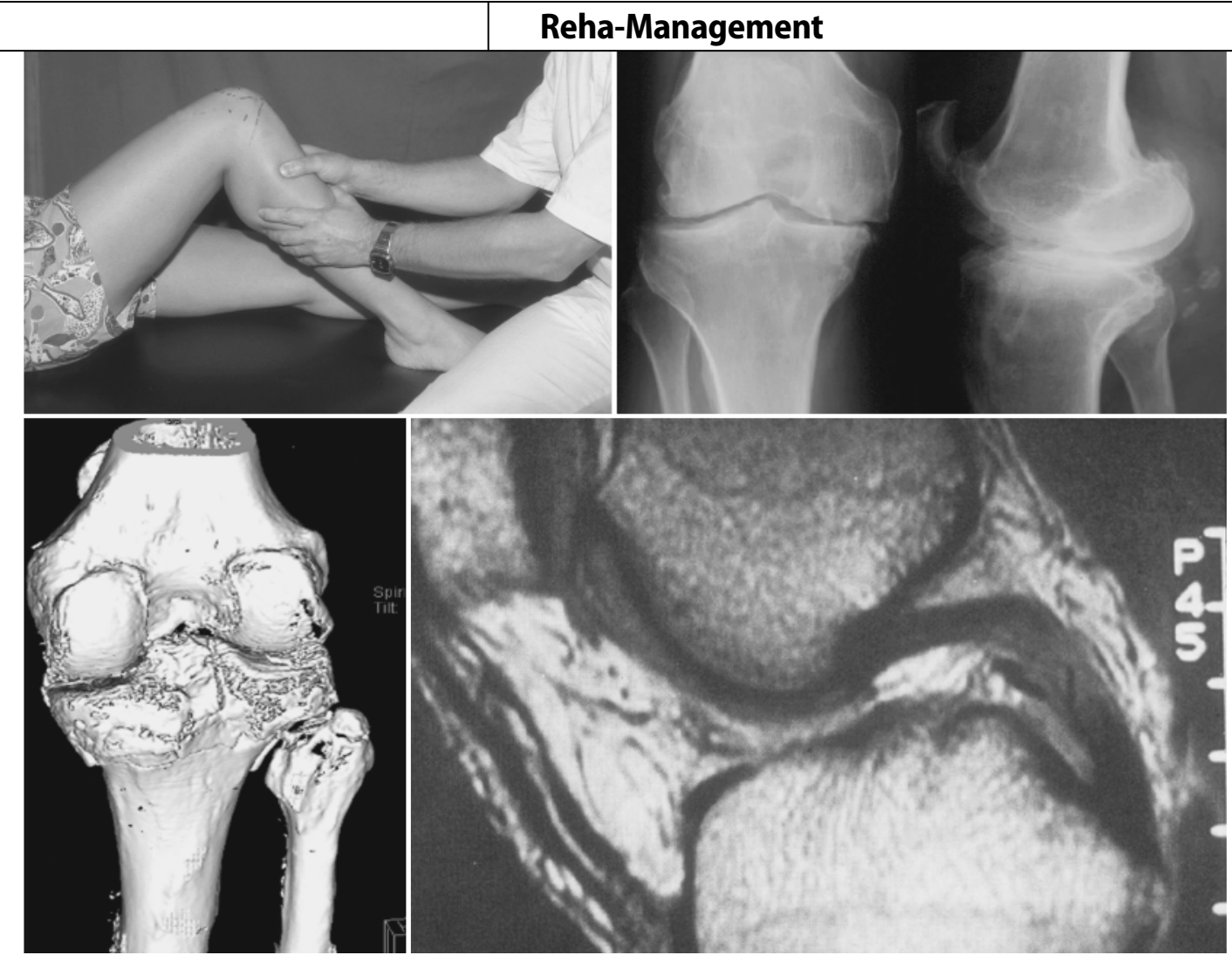

Abb. 14 Ärztliche detaillierte Feststellung der Verletzungsfolgen
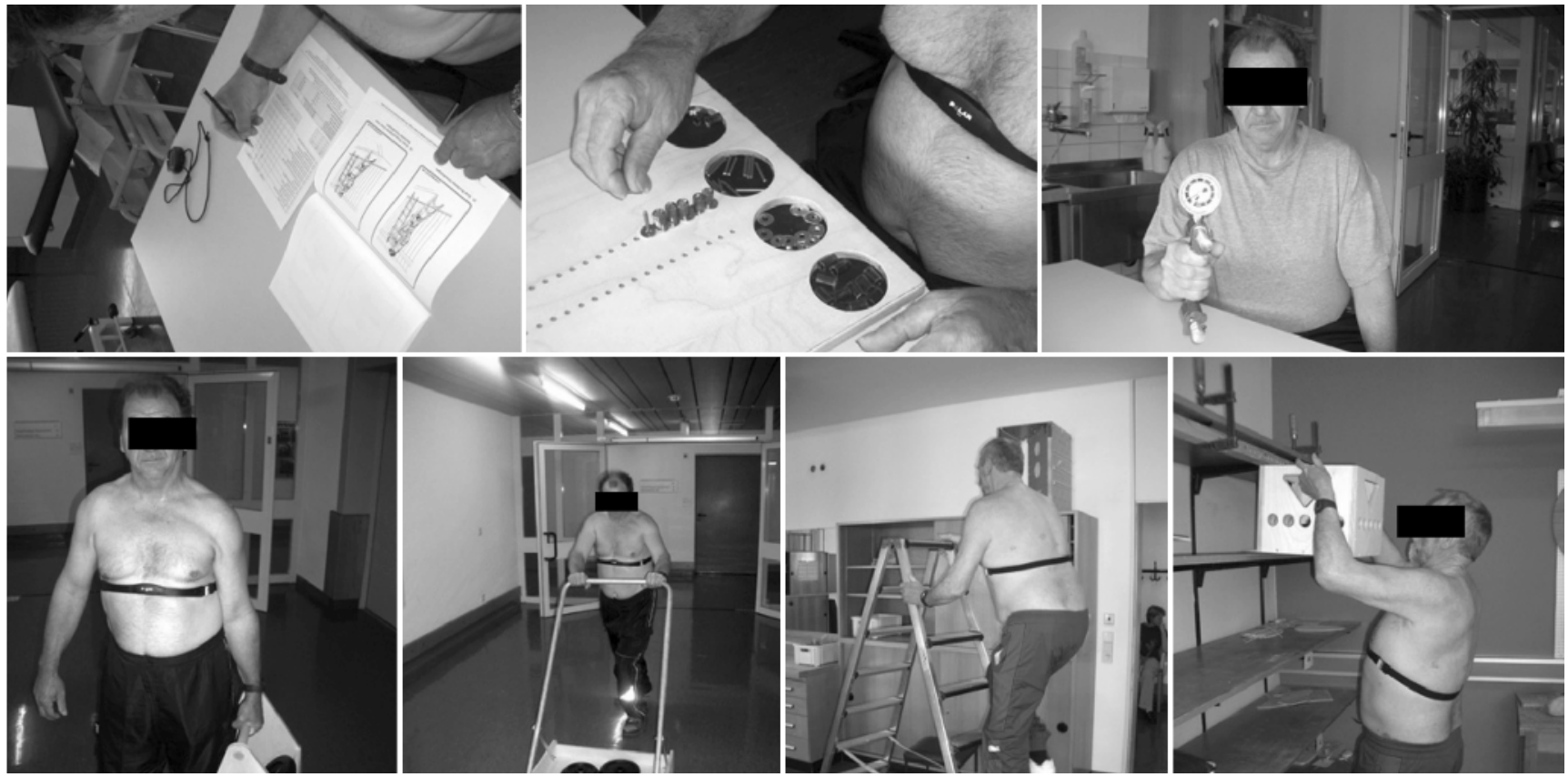

Abb. $3 \Delta$ Evaluation der funktionellen Leistungsfähigkeit

zeichnis sowie eine Stellungnahme des Berufshelfers bezüglich dem Arbeitsplatz und den hier bestehenden Reintegrationsmöglichkeiten benötigt; hierfür steht ein spezielles Formular zur Verfügung.

Am Ende des Verfahrens werden durch das Evaluationsteam unter umfassender Berücksichtigung des Verletzungsmusters, bestehender Defizite und der Mo- tivation ein umfassender Befundbericht, fallweise mit einer Empfehlung weiterer medizinischer Maßnahmen, sowie ein ausführlicher Rehabilitationsplan mit einer Prognose der Reintegrationsfähigkeit und mit Hinweisen für den Berufshelfer erstellt.

An dem Abschlussgespräch nimmt auch der Verletzte teil, zudem besteht die
Möglichkeit einer Teilnahme eines Vertreters des Auftraggebers.

\section{Erste Erfahrungen}

In den BG-Unfallkliniken Ludwigshafen und Tübingen wurden seit dem Start des Projekts im September 2004 bis September 2005 insgesamt 90 dieser um- 

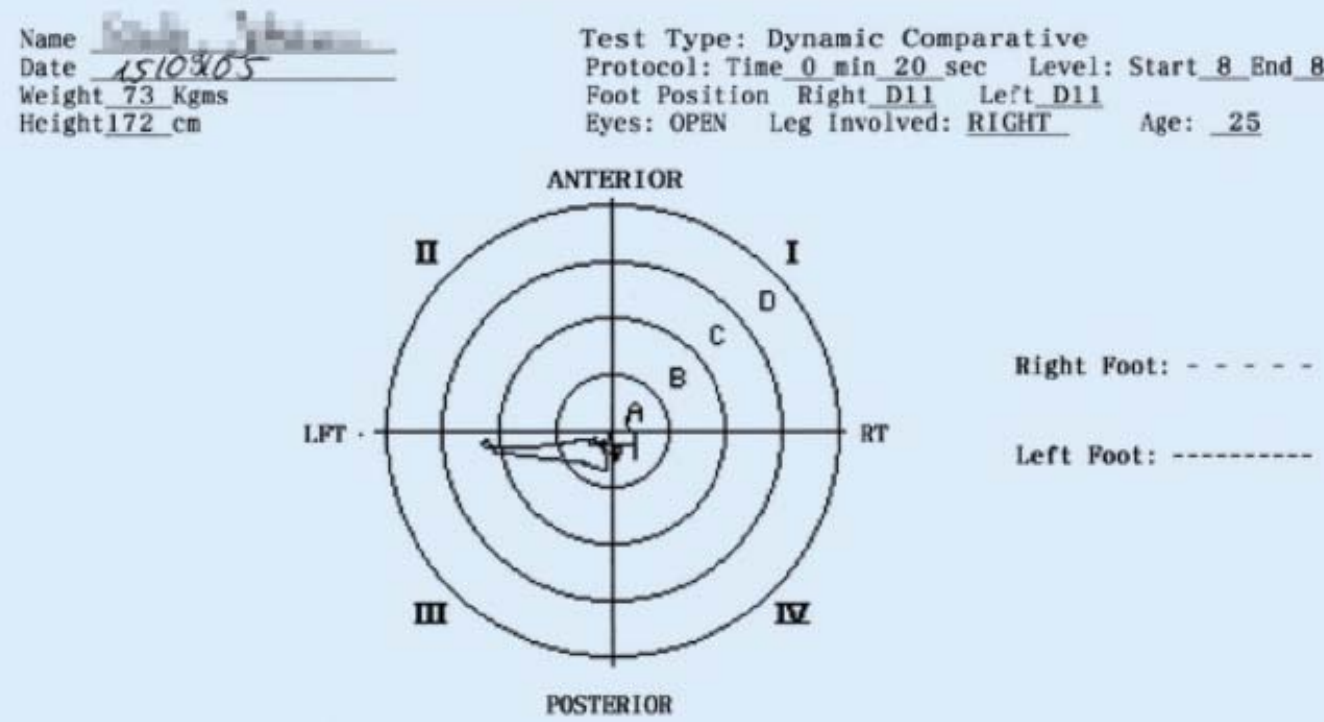

Right Foot: - . - -

Left Foot:

PERCENT

DIFFERENCE
ACTUAL

VALUES

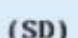

LEFT

(SD)

$59 x$

$35 \%$

$59 \%$
RIGHT

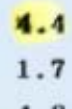

1.9

0.6

2. 2
1.8

1. 1

1. 7
0.2

0.2

0.3

\section{LEGEND:}

OVERAL.L. BALANCB INDEX: Represents the patients ability to control their balance in all directions. High values represent patient had difficulty and further assessment may be needed.

Anterior/Posterior Index:Represents the patients ability to control their balance in front to back direction. High values represent patient had difficulty and further assessment may be needed.

Medial/Lateral Index: Represents the patients ability to control their balance fron side to side. IIigh values represent patient had difficulty and further assessment may be needed. Standard deviation(SD): Represents repeatability in performance. A low SD indicates the range of values, from which the data was calculated, was close together.

Percent Difference: 0 - $10 \pi$ No signifigant difference between extremities. 11 - $25 \%$ Rehabilatation reconnended to improve balance test results.

$>25 \%$ Signifigant functional impairment.

Negative values indicate involved extrenity has better dynanic balance control.

Abb. 24 Ausführliche physiotherapeutische Evaluation. Fortsetzungen auf Seite S114 und S115 fassenden Rehabilitationsabklärungen durchgeführt.

Aus ärztlicher Sicht ist das relativ aufwändige Verfahren positiv zu beurteilen. In aller Regel war es möglich, einen Konsens aller Beteiligten bezüglich des weiteren Verlaufs des Heilverfahrens herbeizuführen, wobei auch meist eine Zustimmung der Verletzten zu erkennen war.

Wesentlich zur Beurteilung des Nutzens des Verfahrens ist für die beteiligten Kliniken die Kenntnis der weiteren schlussendlichen Entwicklung, die zunächst meist nur dem Auftraggeber bekannt ist. Aus ihr ergibt sich für das Eva- luationsteam die Möglichkeit einer Optimierung der Untersuchungen und der Aussage über den zu erwartenden weiteren Verlauf sowie schließlich der Effizienz der ausgesprochenen Empfehlungen.

Um einen ersten Überblick über die letztendlich resultierenden Ergebnisse und die Akzeptanz des neuen Verfahrens bei den Berufsgenossenschaften zu erhalten, wurden von den Ludwigshafener Kollegen Rückmeldebögen an die Auftraggeber versandt. Um eine aussagekräftige Beurteilung zu ermöglichen, wurden dabei nur die länger zurückliegenden Rehabilitationsabklärungen aus dem Zeitraum
September 2004-April 2005 berücksichtigt, da erst nach Ablauf einer gewissen Zeitspanne eine zuverlässige Aussage bezüglich des Erfolgs der empfohlenen Maßnahmen möglich ist.

Zum Zeitpunkt der Auswertung im Oktober 2005 lagen von 37 versandten Anfragen 25 Antworten vor, d. h. die Rücklaufquote lag bei immerhin knapp 70\%.

Die in - Tab. 1 dargestellten Zahlen sind im Sinne einer ersten und vorläufigen Auswertung zu werten, können jedoch durchaus im Sinn eines Trends verstanden werden: Diese ersten Ergebnisse sind durchaus ermutigend. Die Beurtei- 


\section{Reha-Management}

BG Unfallklinik Tubingen

\begin{tabular}{|c|c|c|c|c|}
\hline \multicolumn{5}{|c|}{ Sprunggelenk PLantar-JDorsalflexion (Knieflexion) } \\
\hline Name: & 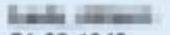 & ID: & & Rechts/Links: 16.09 .200516 .09 .2005 \\
\hline Geburtsdatum: & 21.08 .1949 & Betroff & Rechts & Gruppe 1: \\
\hline $\begin{array}{l}\text { Grosse: } \\
\text { Gewicht: }\end{array}$ & $\begin{array}{l}170 \text { Zentimeter } \\
73 \text { Klogramm }\end{array}$ & $\begin{array}{l}\text { Domina } \\
\text { Arzt: }\end{array}$ & ; Rechts & Gruppe 2: \\
\hline $\begin{array}{l}\text { Geschlecht: } \\
\text { Diagnose: }\end{array}$ & Mannl. & Tester: & Christof Ziegler & \\
\hline
\end{tabular}

Plantarlexoeren

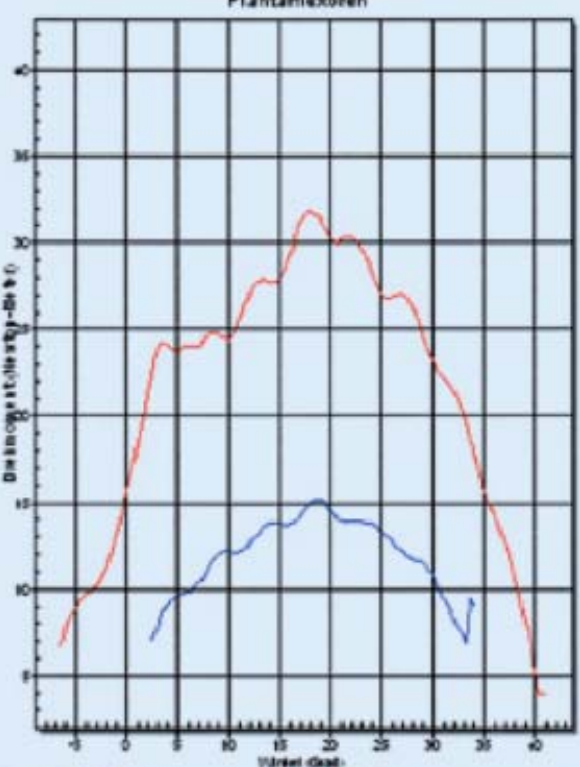

Dowallexoren

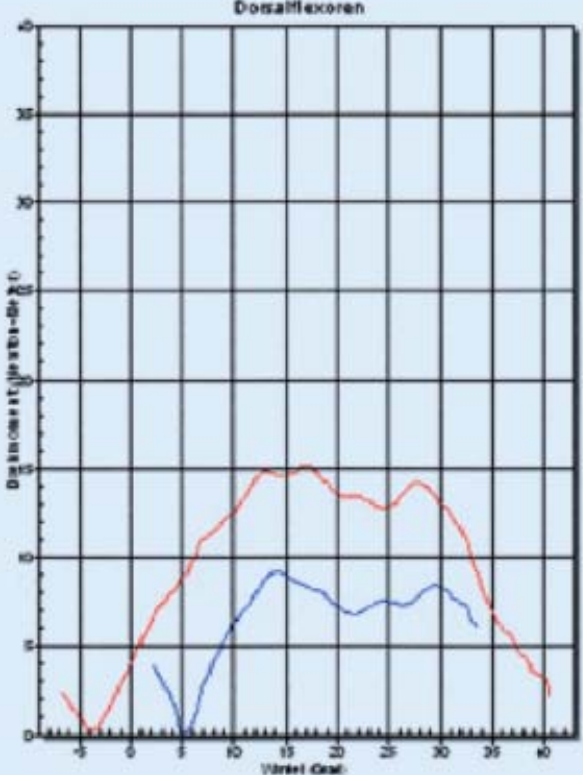

Rechte Seite

unke Seit

\begin{tabular}{|c|c|c|c|c|c|c|c|}
\hline \multirow{2}{*}{$\begin{array}{l}\text { Isokinetisch Kon/Kon } \\
\text { Geschw } 30 / 30 \text { Wdh } 5\end{array}$} & \multicolumn{3}{|c|}{ Plantarflexoren } & \multicolumn{3}{|c|}{ Dorsalflexoren } & \multirow[b]{2}{*}{ Quotient } \\
\hline & Werte & Var & *kg & Werte & Var & $\% k g$ & \\
\hline \multicolumn{8}{|c|}{ Drehmomentmaximum (Newton-Meter -Durchschnittswert) } \\
\hline Rechts & 14 & 0,12 & 18 & 9 & 0,17 & 12 & 70 \\
\hline Links & 30 & 0.07 & 42 & 16 & 0.15 & 24 & 55 \\
\hline Defizit & 55 & & & 42 & & & \\
\hline \multicolumn{8}{|c|}{ Arbeit pro Wiederholung (Newton-Meter - Durchschnittswert) } \\
\hline Rechts & 5 & 0,16 & 6 & 4 & 0,16 & 6 & 75 \\
\hline Uinks & 16 & 0,09 & 24 & $\vec{B}$ & 0,11 & 12 & 50 \\
\hline Defizit & 67 & & & 50 & & & \\
\hline \multicolumn{8}{|l|}{ Bewegungsausmass (Grad) } \\
\hline Rechts & 34 & 0,00 & & 1 & 0.53 & & \\
\hline Links & 41 & 0,00 & & .7 & $-0,04$ & & \\
\hline \multirow{2}{*}{$\begin{array}{l}\text { Isokinetisch Kon/Kon } \\
\text { Geschw } 120 / 120 \text { Wdh } 15\end{array}$} & \multicolumn{3}{|c|}{ Plantarflexoren } & \multicolumn{3}{|c|}{ Dorsalflexoren } & \\
\hline & Werte & Var & $\% \mathrm{~kg}$ & Werte & Var & $\% \mathrm{~kg}$ & Quotient \\
\hline \multicolumn{8}{|c|}{ Initialdrehmoment (Newton-Meter - Durchschnittswert) } \\
\hline Rechts & 8 & 0,00 & 12 & 9 & 0.00 & 12 & 117 \\
\hline Links & 8 & 0,00 & 12 & 8 & 0.00 & 12 & 100 \\
\hline Defizit & 0 & & & -14 & & & \\
\hline \multicolumn{8}{|l|}{ Ermüdungsindex } \\
\hline Rechts & 7 & 0,00 & & 29 & 0,00 & & \\
\hline Uniks & 22 & 0,00 & & 3 & 0,00 & & \\
\hline \multicolumn{8}{|c|}{ Gesamtarbeit (Newton-Meter) } \\
\hline Rechts & 30 & 0,00 & 42 & 23 & 0,00 & 33 & 77 \\
\hline Links & 47 & 0,00 & 66 & 27 & 0,00 & 36 & 57 \\
\hline Defizit & 37 & & & 15 & & . & 103 \\
\hline
\end{tabular}

Abb. 2 - Fortsetzung 1

HUMAC3 for Wndows Version: 4.3.8 Cepyright Computer Sports Medicine, Inc., 1982-2003

lung und damit die Akzeptanz dieses im Rahmens eines Modellprojekts neuartigen Verfahrens sind sowohl bei den Berufsgenossenschaften als auch bei den Versicherten überwiegend positiv. In der deutlichen Mehrzahl der Fälle äußerten sich die Versicherten zufrieden oder sehr zufrieden. Manche zeigten sich allerdings mit dem Verfahren insgesamt oder auch teilweise nicht zufrieden. Dies kann u. a. darauf zurückgeführt werden, dass ihre
Vorstellungen nicht immer verwirklicht werden konnten.

Die gegebenen Empfehlungen an die Versicherungsträger wurden mit 17 positiven Antworten von diesen als für die Versicherten überwiegend erfolgreich angesehen. Bei nicht erfolgreichem weiterem Verlauf wurden von den Sachbearbeitern als möglicher Grund eine mangelnde Bereitschaft der Versicherten zur Mitarbeit und ein Misstrauen gegenüber ärztlichen Diagnosen und Ratschlägen angeführt.

Relativ hoch erscheint der Anteil von Versicherten, bei denen die Arbeitsfähigkeit (zunächst) nicht wiederhergestellt werden konnte. Er ist darauf zurückzuführen, dass in 4 der 11 Fälle eine berufliche Umorientierung in die Wege geleitet werden musste, bei 7 dieser Versicherten kam es aus unterschiedlichen Grün- 


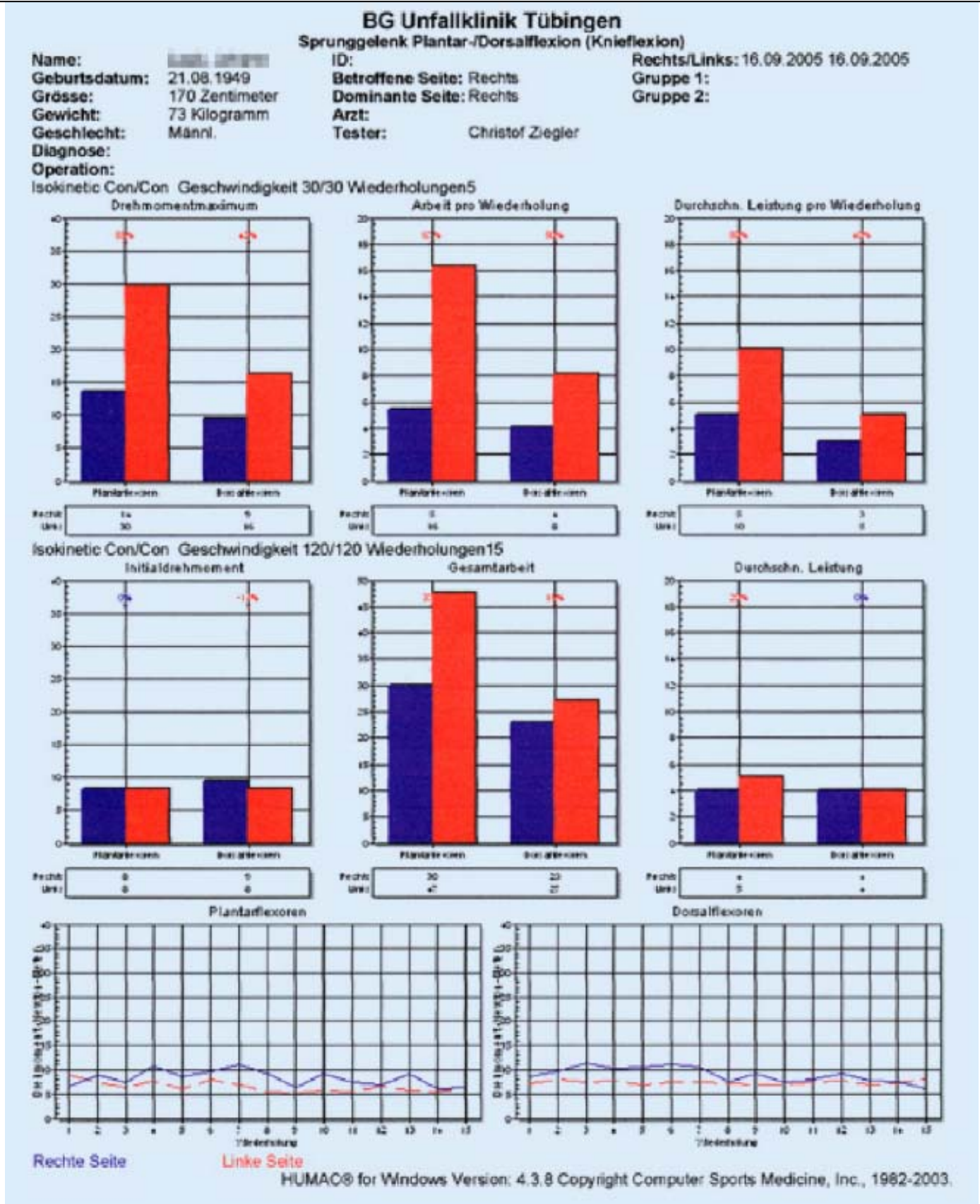

Abb. $2 \triangleleft$ Fortsetzung 2

den ohne Wiedererlangung der Arbeitsfähigkeit zu einer Berentung.

Abschließend bleibt die Feststellung, dass nach ersten Beurteilungen und Auswertungen das Angebot des Projekts der Rehabilitationsabklärung von den Berufsgenossenschaften positiv angenommen wurde und auch von den beteiligten Kliniken aufgrund der gewonnenen Erfahrungen für sinnvoll erachtet wird.

In den meisten Fällen konnten durch das Kompetenzteam bei problematischen Heilverläufen richtungweisende Impulse gegeben werden, die häufig zur baldigen Rückkehr der Versicherten an ihren Ar- beitsplatz oder zur Einleitung weiterer berufsbezogener Maßnahmen und zu einem zufrieden stellenden Abschluss des Heilverfahrens führten.

Es ist vorgesehen, diese ersten Erfahrungen durch weitere Auswertungen zu vertiefen.

Weitere Hinweise und Einzelheiten über das Projekt können auf der Homepage der beiden Kliniken eingesehen werden:

- http://www.bgu-lu.de bzw.

- http://www.bgu-tuebingen.de,

jeweils unter dem Link „BG-Portal“ $\rightarrow$ “ spezielle Leistungsangebote“.

\section{Korrespondierender Autor}

Dr. M. Vogt

Klinik für Unfall- und Wiederherstellungschirurgie, BG-Unfallklinik

Schnarrenbergstraße 95, 72076 Tübingen m.vogt@bgu-tuebingen.de

Interessenkonflikt. Es besteht kein Interessenkonflikt. Der korrespondierende Autor versichert, dass keine Verbindungen mit einer Firma, deren Produkt in dem Artikel genannt ist, oder einer Firma, die ein Konkurrenzprodukt vertreibt, bestehen. Die Präsentation des Themas ist unabhängig und die Darstellung der Inhalte produktneutral. 